\title{
Ultrasound-Based Multimodal Imaging Predicting Ischemic-Type Biliary Lesions After Living-Donor Liver Transplantation
}

\author{
Jin-qiao Liu \\ Wen-juan Chen (D) \\ Meng-jie Zhou \\ Wen-feng Li \\ Ju Tang
}

Department of Ultrasound, Hunan Children's Hospital, Changsha City, Hunan Province, People's Republic of China
Correspondence: Wen-juan Chen Department of Ultrasound, Hunan Children's Hospital, No. 86 Ziyuan Road, Changsha City, Hunan Province, 410007,

People's Republic of China

Tel + 86-13707484196

Email cwj299979@aliyun.com
Background: Ischemic-type biliary lesions (ITBL) are accepted as the most incomprehensible biliary complications after living-donor liver transplantation (LDLT). Early predicting the development of ITBL in pediatric patients permits more preventive strategies. However, few studies have focused on the early prediction of ITBL.

Objective: This study aimed to establish a nomogram including ultrasound-based multimodal imaging to predict ITBL in children with biliary atresia (BA) within 2 years after receiving LDLT. Methods: The records of 94 BA children with at least one year of follow-up after LDLT were reviewed retrospectively. They were randomly divided into a training cohort for constructing a nomogram $(n=64)$ and a validation cohort $(n=30)$. In the training cohort, patients diagnosed as ITBL were included in the ITBL group and those without any vascular and biliary complication were included in the non-ITBL group. Multivariate Cox regression was used for the establishment of the nomogram in predicting the risk of ITBL within 2 years post-LDLT. The discrimination and calibration of the nomogram were internally and externally validated. The performances of the nomogram and the individual components were compared by the area under the curve (AUC) of receiver operating characteristic (ROC) curve.

Results: In the training cohort, 18 BA children were included in the ITBL group and 46 were in the non-ITBL group. Last pediatric end-stage liver disease (PELD) score, gammaglutamyl transpeptidase (GGT), resistive index (RI), and liver stiffness measurement (LSM) were the independent predictors for the development of ITBL within 2 years post-LDLT. The nomogram incorporating these independent predictors showed good discrimination and calibration by the internal and external validation. Its performance was better than any individual component in predicting the prognosis $(P<0.05)$.

Conclusion: The established nomogram may be used to predict the risk of ITBL within 2 years post-LDLT in BA children.

Keywords: ischemic-type biliary lesions, living-donor liver transplantation, pediatric patients, biliary atresia, color Doppler flow imaging, liver stiffness measurement

\section{Introduction}

Living-donor liver transplantation (LDLT) has been widely accepted as the treatment for infantile biliary atresia (BA) and other end-stage liver diseases in children. ${ }^{1,2}$ With the further understanding of biliary complications and great improvements in the surgical techniques, the incidence of anastomotic complications has declined. ${ }^{3}$ Ischemic-type biliary lesions (ITBL) are defined by nonanastomotic intra- or extrahepatic bile duct strictures after liver transplantation, when there is no evidence of hepatic artery stenosis or thrombosis, portal 
thrombosis, organ rejection, primary sclerosing cholangitis, or other causes of bile duct damage. ${ }^{4}$ It is considered to be one of the most troublesome biliary complications after liver transplantation due to the characteristics of nonanastomotic intrahepatic strictures secondary to microangiopathy. ${ }^{4-7}$ The reported incidence of ITBL is $5-32 \%$ that should not be ignored. ${ }^{8,9}$ It increases the risk of graft failure and reduces the overall graft survival. ${ }^{10}$ The pathogenesis of ITBL remains unclear. Possible causative factors include ischemia and ischemia-reperfusion injury, cytotoxic effects of bile salts, and immune-mediated injury, etc. ${ }^{6,11-14}$ However, the insufficient blood supply to the hepatic artery (thrombosis or stenosis) caused by any reason is one of the recognized and important factors. ${ }^{15}$ Since the interrupted tiny arterioles surrounding the biliary tree contribute to the decreased ischemic tolerance of liver graft, ITBL may develop even if only a mild alteration in the hepatic artery blood flow, which is difficult to detect by color Doppler flow imaging (CDFI). Currently, ultrasound examination is a routine monitoring option for follow-up after liver transplantation (gray-scale ultrasound for bile duct and CDFI for hepatic artery blood flow). ${ }^{16,17}$ However, it is difficult to detect ITBL in time in postoperative monitoring to conduct an early treatment which helps prevent bile duct obstruction. We still lack a fast, convenient, and sensitive means for ITBL screening. Previous studies mainly focused on the hemodynamic changes of hepatic vasculature and liver parenchymal elasticity after liver transplantation. ${ }^{12,18-20}$ Few series have focused on the early prediction of ITBL. Studies have found that persistent chronic cholestatic liver injury due to ITBL may cause hepatic fibrosis, and liver stiffness measurement (LSM) by elastography has become a reliable and popular noninvasive method in assessing hepatic fibrosis and cholestasis, which has been widely equipped in ultrasound instruments. $6,18,19,21-23$

We therefore conducted a study for analyzing the early characteristics of LSM values and serological changes detected during the follow-up in children with ITBL. The aim of this study was to establish a nomogram to predict ITBL in BA children after LDLT.

\section{Materials and Methods}

\section{Population}

This retrospective cohort study was conducted following the Declaration of Helsinki and the Declaration of Istanbul. All procedures were approved by the ethical review committee of Hunan Children's Hospital (HCHLL-2020-99). Informed consents were obtained from their parents or legal guardians, and all living donor organs were donated voluntarily with written informed consent.

Between May 2017 and December 2020, the medical records of 116 pediatric patients ( $<12$ years) with BA underwent LDLT and their follow-ups were reviewed in this study. The inclusion criteria were children with good postoperative liver function and at least one year of follow-up. The exclusion criteria were as follows: (a) any reason that led to a prolonged intensive care unit stay; (b) vascular complications, such as thrombosis or stricture, were diagnosed; (c) bile duct leakage or anastomotic stricture was found; and (d) early rejection episodes within one-month after LDLT was diagnosed. At last, 94 patients were enrolled, and the eligible patients were divided into a training cohort for constructing a nomogram $(n=64)$ and a validation cohort $(n=30)$ using a random number table (Figure 1).

Piggyback liver transplantation was performed for all children. Donor liver graft types were the left lateral segment. Preoperative magnetic resonance cholangiopancreatography (MRCP) was performed to evaluate the donor biliary anatomy and detect asymptomatic biliary abnormalities. The reconstruction procedures were performed under an operating microscope and varied according to the cases. Basically, arterial anastomoses were performed with interrupted stitches. A continuous suture on the posterior wall and interrupted stitches on the anterior wall were performed in all venous systems. The bile duct was reconstructed by Roux-en-Y hepaticojejunostomy with interrupted sutures and no stent was ever used. Postoperative ultrasound and MRCP were performed to check the vascular flow and the status of the biliary system.

Each patient received perioperative antiinflammation, anticoagulation, and anti-rejection prophylaxis. Biopsy-proved rejection was treated with triple immunosuppression therapy including mycophenolate mofetil, prednisolone, and tacrolimus. Eight variables were analyzed which were related to the recipients' demographics and operative characteristics, including age at LDLT, gender, weight at LDLT, last pediatric end-stage liver disease (PELD) score prior to LDLT, Kasai portoenterostomy (KPE) history, graft-torecipient weight ratio (GRWR), cold ischemia time, and intraoperative blood loss volume. 


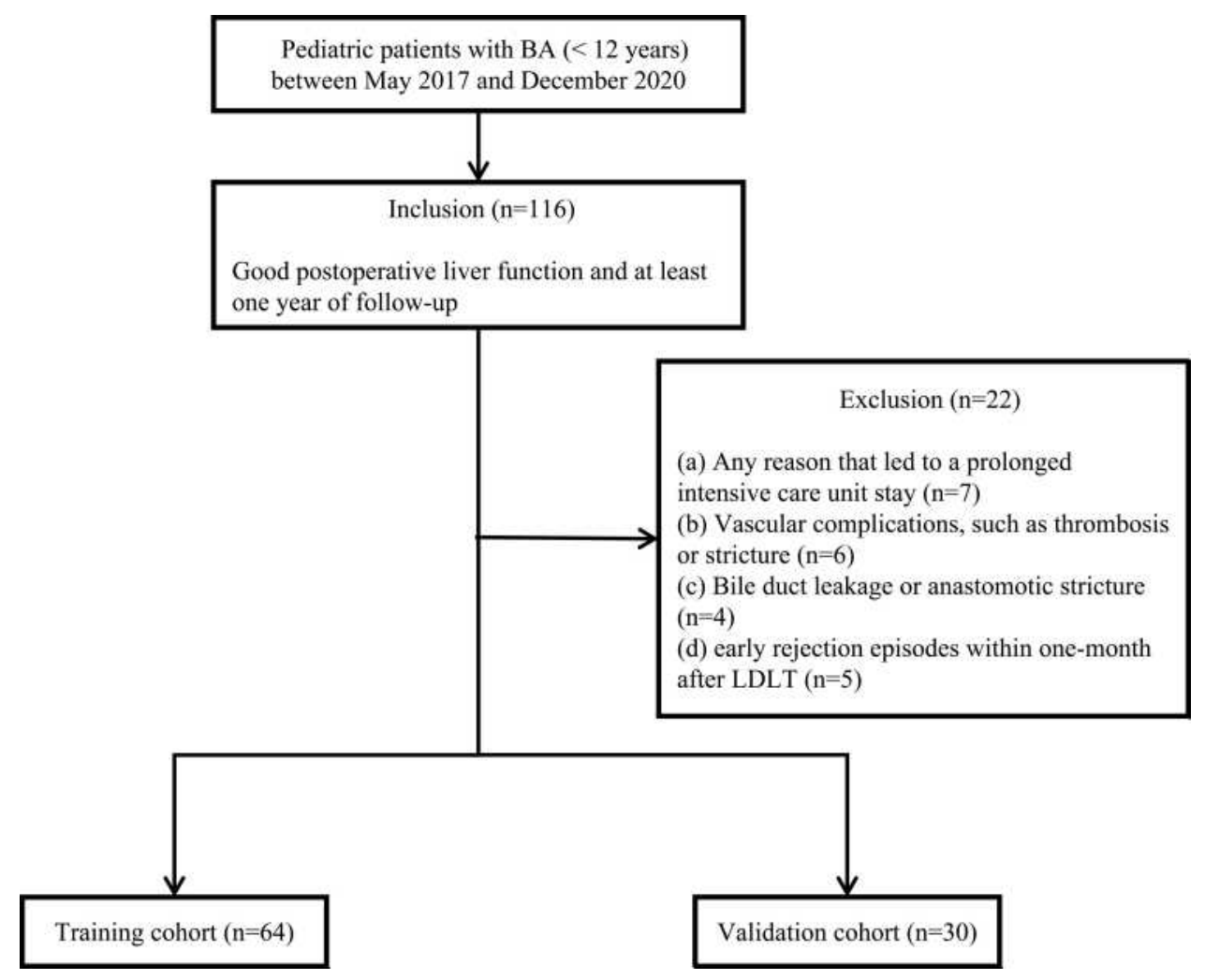

Figure I Flow chart of patient selection for training and validation cohorts. Abbreviations: BA, biliary atresia; LDLT, living-donor liver transplantation.

\section{Assessment of Ultrasound-Based Multimodal Imaging}

LSM obtained by shear wave elastography (SWE) has been routinely performed since 2017 . The children were assessed in the supine position with peaceful breathing after a 6-hour fast. An Aixplorer ultrasound system (SuperSonic Imagine, Aix-en-Provence, France) was performed by two sonographers with more than 10 years of experience in abdominal ultrasound and more than 3 years of experience in elastography techniques. After routine abdominal examinations, the filling of the graft hepatic artery and portal vein was observed in CDFI mode. The peak systolic velocity (PSV), end-diastolic velocity (EDV), resistive index (RI), and pulsation index (PI) of hepatic artery and portal vein velocity (PVV) were measured by pulsed Doppler.

SWE was performed through the right intercostal space. The measurement depth was between 2.5 and $5.5 \mathrm{~cm}$ below the skin surface. The range of the SWE sampling frame $(2 * 2 \mathrm{~cm})$ was set $1 \mathrm{~cm}$ below the liver capsule of the right anterior lobe while large vessels, intrahepatic biliary tracts, and gallbladder were avoided. A circular region of interest (ROI) $(10 \mathrm{~mm}$ in diameter) was positioned in the sampling frame. A valid SWE was achieved when most of the ROI $(>90 \%)$ was filled with homogeneous color. Five valid measurements were recorded for each child within 5 minutes, and the median value was considered representative of the elastic modulus of the liver and expressed in kilopascals ( $\mathrm{kPa}$ ) (Figure 2).

\section{Study Protocol}

After discharge, the pediatric recipients were followed up weekly within 3 months, biweekly within 3 to 6 months, monthly within 6 to 12 months, and as needed after one year. Ultrasound assessment and laboratory data including aspartate aminotransferase (AST), alanine aminotransferase (ALT), total bilirubin (TBil), direct bilirubin (DBil), gammaglutamyl transpeptidase (GGT), as well as alkaline phosphatase (ALP) were recorded one-month post-LDLT.

ITBL was diagnosed by ultrasound or MRCP, and confirmed and treated using percutaneous transhepatic cholangiography (PTC). PTC was performed using a fineneedle puncture technique. Contrast agent was infused through the needle to obtain cholangiography. Treatments such as drainage were considered according to the findings of cholangiography. Complications such as hemorrhage 


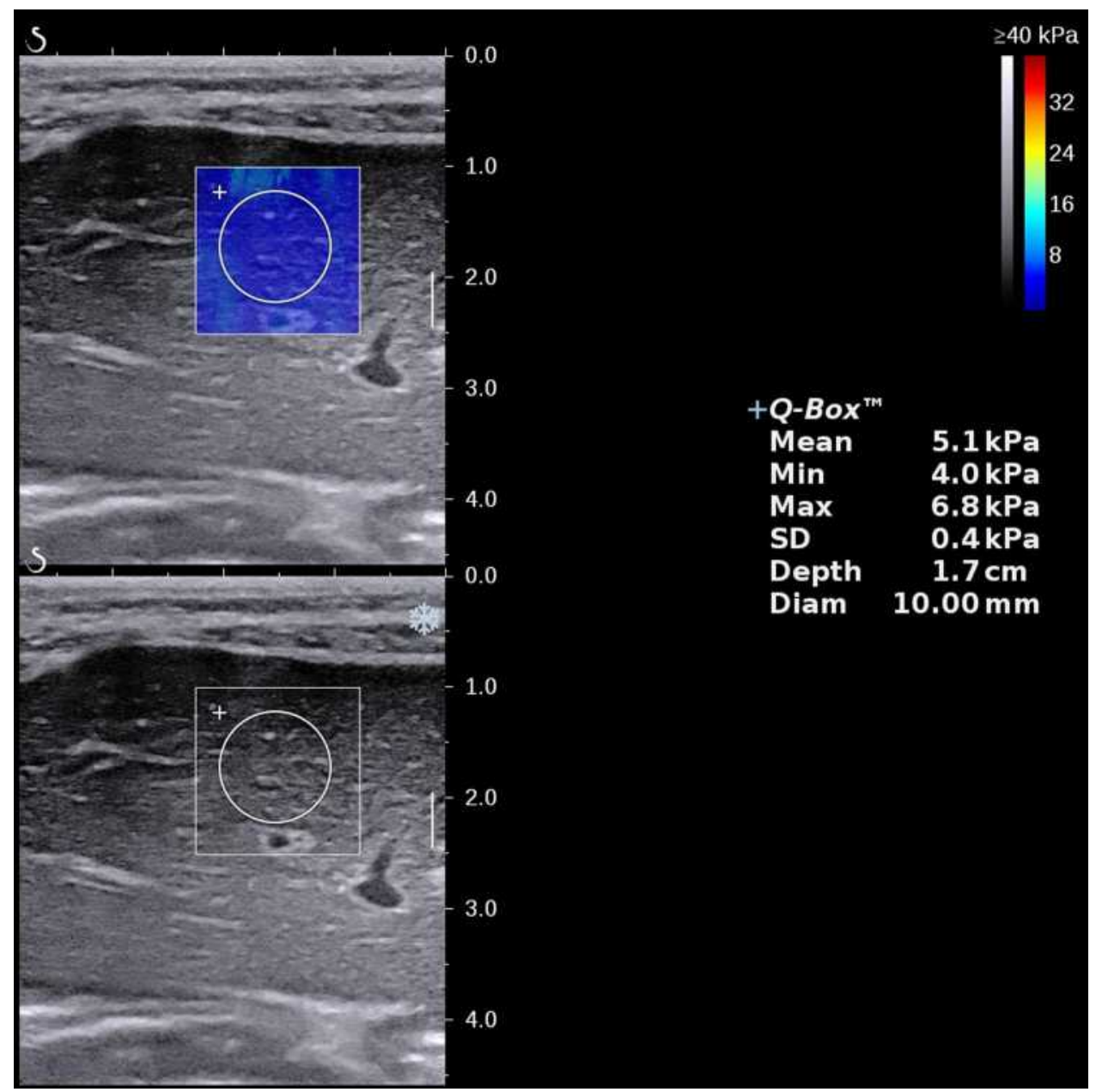

Figure 2 LSM obtained by SWE in BA child underwent LDLT. The SWE showed a stiffness color map (top) and a grayscale image (bottom). The mean LSM value in the ROI was $5.1 \mathrm{kPa}$.

Abbreviations: LSM, liver stiffness measurement; SWE, shear wave elastography; BA, biliary atresia; LDLT, living-donor liver transplantation; ROI, region of interest.

and cholangitis were not found. Patients with ITBL during follow-up were included in the ITBL group. Those without any vascular and biliary complications during follow-up were included in the non-ITBL group.

\section{Statistical Analysis}

The incidence of ITBL during follow-up was analyzed by Kaplan-Meier (KM) method. The indicators of liver function and ultrasound-based multimodal imaging at onemonth post-LDLT were compared between the two groups to screen the potential variables for the prediction of ITBL. The inter-observer reliability of LSM values was studied by intraclass correlation coefficient (ICC) (ICC $>0.75$ for good repeatability). Independent sample $t$-test and MannWhitney $U$-test were used for the comparison of normally distributed and non-normally distributed variables, respectively. Fisher exact test was used for the comparison of categorical variables. The variables that were significant at $P<0.05$ in the comparison included in the multivariate Cox proportional hazards regression model.

A nomogram based on the independent predictors was developed to predict the risk of ITBL after LDLT. It was internally validated in the training cohort and externally validated in the validation cohort. Both discrimination and calibration were evaluated using bootstrapping validation (1000 bootstrap resamples) to obtain relatively unbiased estimates of the performance of the model. The discrimination of the nomogram was evaluated by the area under the curve (AUC) of receiver operating characteristic (ROC) curve (AUC $>0.75$ indicating good discrimination). A calibration curve, which plotted the average KaplanMeier estimate against the corresponding prognosis predicted by the nomogram, combined with the HosmerLemeshow (HL) test were utilized to evaluate the calibration 
of the nomogram. IBM SPSS Statistics 22.0 (IBM Corp., Armonk, NY, United States), R package version 3.6.2 and MedCalc (Version 22.0.1; MedCalc Software, Ostend, Belgium) were used for statistical analyses.

\section{Results}

\section{Characteristics and Incidences of ITBL} After LDLT in the Training and Validation Cohorts

The characteristics of the training and validation datasets were comparable in both cohorts, justifying their use as training and validation datasets. There was no significant difference in the incidence of ITBL within 2 years postLDLT between the training (28.1\%) and validation datasets $(26.7 \%)$ (Figure 3).

\section{Baseline Characteristics Between the ITBL Group and the Non-ITBL Group}

Of the 64 pediatric patients in the training cohort [ 30 boys and 34 girls, age at LDLT $6(2,9)$ years], 18 were included in the ITBL group and 46 were in the non-ITBL group. Comparisons of the baseline characteristics between the two groups are shown in Table 1. The last PELD score in the ITBL group was higher than in the non-ITBL group $(P<0.05)$. There was no statistically significant difference in other characteristics between the two groups (all $P>0.05)$.

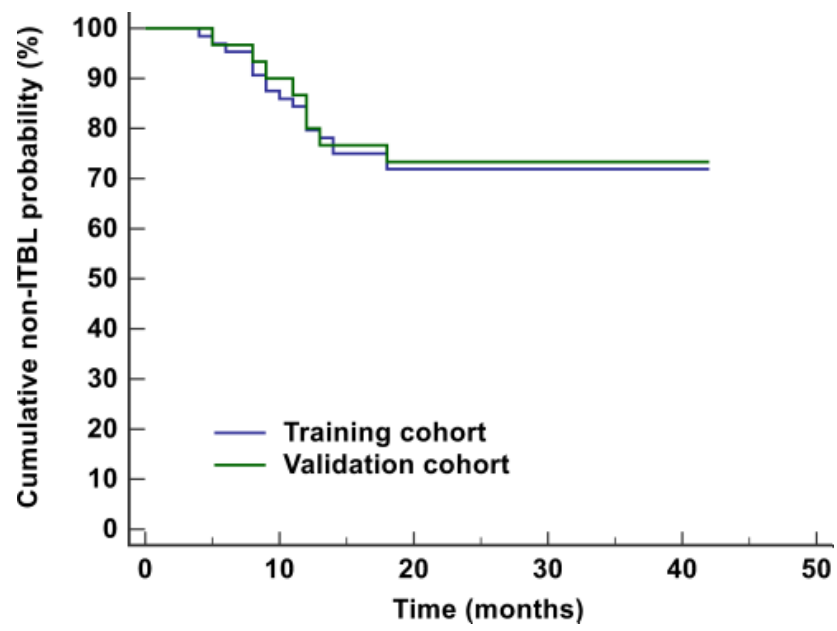

Figure 3 Kaplan-Meier analysis for the incidence of ITBL in the training cohort and validation cohort within 2 years after LDLT. The incidence of ITBL in the training cohort and validation cohort were $28.1 \%$ and $26.7 \%$, respectively.

Abbreviations: ITBL, ischemic-type biliary lesions; LDLT, living-donor liver transplantation.
Table I Comparison of the Baseline Characteristics Between the ITBL Group and the Non-ITBL Group

\begin{tabular}{|c|c|c|c|c|}
\hline \multicolumn{2}{|c|}{ Characteristics } & $\begin{array}{l}\text { ITBL Group } \\
(n=18)\end{array}$ & $\begin{array}{c}\text { Non-ITBL } \\
\text { Group }(n=46)\end{array}$ & $P$ \\
\hline \multicolumn{2}{|c|}{ Age at LDLT (year) } & $6.5(2,9)$ & $5.5(3,7.5)$ & $0.875^{\#}$ \\
\hline Gender & Male & $10(55.6 \%)$ & $21(45.7 \%)$ & $0.581^{\$}$ \\
\hline [n (\%)] & Female & $8(44.4 \%)$ & $25(54.3 \%)$ & \\
\hline \multicolumn{2}{|c|}{ Weight at LDLT $(\mathrm{kg})$} & $8.23 \pm 2.61$ & $9.4 I \pm 4.63$ & $0.313^{*}$ \\
\hline \multicolumn{2}{|c|}{ Last PELD score } & $15.33 \pm 4.30$ & $12.59 \pm 4.00$ & $0.019 *$ \\
\hline \multicolumn{2}{|c|}{ KPE history [n (\%)] } & 15 (83.3\%) & 42 (91.3\%) & $0.391^{\$}$ \\
\hline \multicolumn{2}{|c|}{ GRWR (\%) } & $2.59 \pm 0.77$ & $2.75 \pm 0.80$ & $0.487^{*}$ \\
\hline \multicolumn{2}{|c|}{$\begin{array}{l}\text { Cold ischemia time } \\
(\mathrm{min})\end{array}$} & $48(34,63)$ & $53(46,61)$ & $0.292^{\#}$ \\
\hline \multicolumn{2}{|c|}{$\begin{array}{l}\text { Intraoperative } \\
\text { blood loss volume } \\
(\mathrm{mL})\end{array}$} & $252.78 \pm 92.87$ & $262.67 \pm 77.00$ & $0.664^{*}$ \\
\hline
\end{tabular}

Notes: *For independent sample $t$-test, ${ }^{\$}$ for Fisher exact test, ${ }^{\#}$ for Mann-Whitney U-test.

Abbreviations: ITBL, ischemic-type biliary lesions; LDLT, living-donor liver transplantation; PELD, last pediatric end-stage liver disease; KPE, Kasai portoenterostomy; GRWR, graft-to-recipient weight ratio.

\section{Comparisons of the Liver Function and Ultrasound-Based Multimodal Imaging at One-Month Post-LDLT Between the Two Groups}

The ICC for the LSM values was 0.828 , indicating good interobserver reliability. At one postoperative month, the ALP, GGT, RI, and LSM in the ITBL group were higher than those in the non-ITBL group $(\mathrm{P}<0.05)$. The PSV in the ITBL group was lower than the non-ITBL group $(P<0.05)$ (Table 2).

\section{Independent Predictors Associated with ITBL Within 2 Years Post-LDLT}

Multivariate Cox regression further revealed that last PELD score, GGT, RI, and LSM were the independent predictors for the development of ITBL within 2 years post-LDLT (Figure 4). A nomogram incorporating these independent predictors was constructed to predict the risk of ITBL within 2 years post-LDLT (Figure 5). Each predictor was assigned a number with the weight equal to the hazard ratio from the multivariate Cox model and the estimated probability of ITBL in a child was determined by adding the scores of each predictor. 
Table 2 Comparison of the Liver Function and UltrasoundBased Multimodal Imaging at One-Month Post-LDLT Between the Two Groups

\begin{tabular}{|l|c|c|c|}
\hline Variables & $\begin{array}{c}\text { ITBL Group } \\
(\mathbf{n}=18)\end{array}$ & $\begin{array}{c}\text { Non-ITBL Group } \\
(\mathbf{n}=\mathbf{4 6})\end{array}$ & $\boldsymbol{P}$ \\
\hline Liver function & & & \\
ALT (IU/L) & $26.50 \pm 3.24$ & $25.89 \pm 6.08$ & $0.604^{*}$ \\
AST (IU/L) & $28.22 \pm 3.04$ & $26.43 \pm 4.26$ & $0.110^{*}$ \\
TBil (mg/dL) & $16.53(14.17,20.16)$ & $14.56(12.27,18.49)$ & $0.160^{\#}$ \\
DBil (mg/dL) & $5.39(4.75,6.20)$ & $5.27(3.98,6.10)$ & $0.586^{\#}$ \\
ALP (IU/L) & $94.78 \pm 11.74$ & $89.87 \pm 10.65$ & $0.037^{*}$ \\
GGT (IU/L) & $35(24,44.75)$ & $27(22.75,35.25)$ & $0.022^{\#}$ \\
\hline Ultrasound-based & & & \\
multimodal imaging & & & \\
PSV (cm/s) & $55.11 \pm 9.47$ & $62.54 \pm 13.96$ & $0.042^{*}$ \\
EDV (cm/s) & $15.91 \pm 6.60$ & $16.50 \pm 5.01$ & $0.735^{*}$ \\
RI & $0.66 \pm 0.15$ & $0.58 \pm 0.11$ & $0.013^{*}$ \\
PI & $1.03 \pm 0.32$ & $1.05 \pm 0.27$ & $0.865^{*}$ \\
PVV (cm/s) & $48.50 \pm 14.65$ & $50.39 \pm 13.94$ & $0.640^{*}$ \\
LSM value (kPa) & $6.25(5.78,8.50)$ & $5.45(4.65,6.73)$ & $0.002^{\#}$ \\
\hline
\end{tabular}

Notes: *For independent sample $t$-test, ${ }^{\#}$ for Mann-Whitney U-test.

Abbreviations: LDLT, living-donor liver transplantation; ITBL, ischemic-type biliary lesions; ALT, alanine amino-transferase; AST, aspartate aminotransferase; TBil, total bilirubin; DBil, direct bilirubin; ALP, alkaline phosphatase; GGT, gammaglutamyl transpeptidase; PSV, peak systolic velocity; EDV, end diastolic velocity; RI, resistive index; PI, pulsation index; PVV, portal vein velocity; LSM, liver stiffness measurement.

\section{Internal and External Validation of the Nomogram}

In the internal validation, the AUC of the nomogram was 0.888 (95\% CI: 0.784 to 0.953$)$, and a similar concordance index $(0.862,95 \% \mathrm{CI}$ : 0.748 to 0.912$)$ was acquired after 1000 bootstrapping, which indicated good discrimination $(>0.75)$ (Figure 6A). The calibration plot graphically showed that the nomogram-predicted prognosis and actual prognosis estimated using $\mathrm{KM}$ analysis agreed well in the training cohort (Figure 6B). The HL test yielded no significant difference between the predicted and actual prognosis $(P=0.258)$, suggesting good fitting of the nomogram. In the external validation using the validation datasets, the nomogram also displayed good discrimination with an AUC of 0.857 (Figure 6C). Good calibration was also demonstrated by a non-statistical significance obtained in the HL test $(\mathrm{P}=0.178)$, as displayed by the calibration plot (Figure 6D).

The performances of the nomogram and its components were analyzed by ROC curves (Figure 7). The accuracies of last PELD score (AUC: 0.670, 95\% CI: 0.541 to 0.783 ), GGT (AUC: $0.702,95 \% \mathrm{CI}: 0.575$ to 0.810), RI (AUC: 0.646 , 95\% CI: 0.517 to 0.762 ), and LSM (AUC: $0.757,95 \%$ CI: 0.633 to 0.855 ) were lower than that of the nomogram (all $P<0.05$ ). It indicated that except for the nomogram, each predictor could not accurately predict the development of ITBL within 2 years post-LDLT.

\section{Discussion}

ITBL after LDLT, which is accepted as the most incomprehensible type of biliary complications, is associated with a marked decrease in graft survival. ${ }^{6}$ Early predicting the development of ITBL after LDLT in pediatric patients permits more specific preventive and therapeutic strategies. In this study, with the liver function and ultrasoundbased multimodal imaging at one-month post-LDLT, the development of ITBL within 2 years post-LDLT was associated with the features including higher last PELD score, GGT, RI, and LSM. The developed nomogram showed

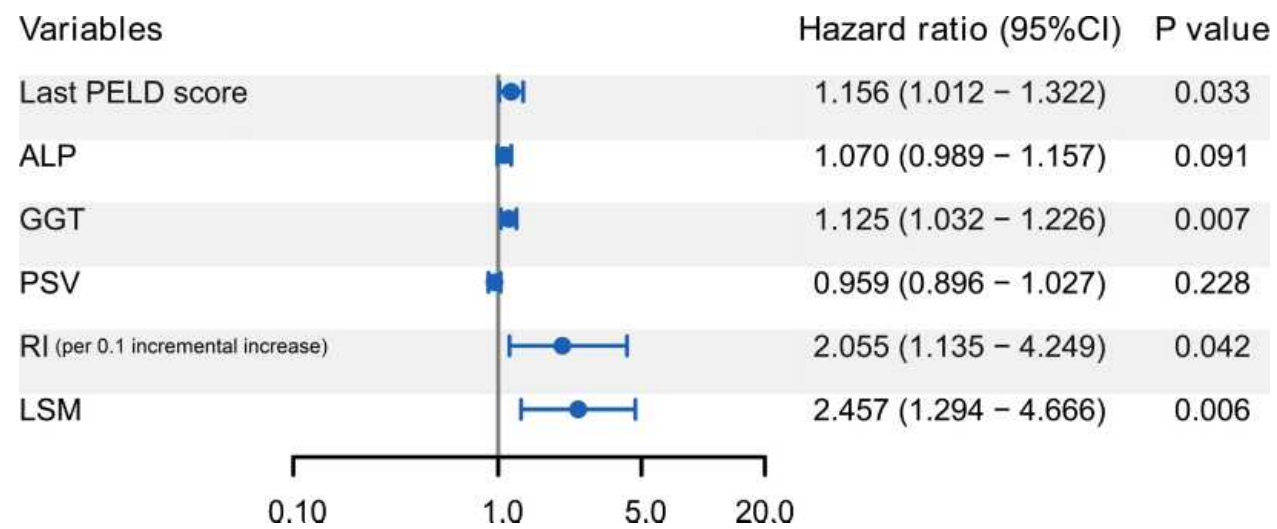

Figure 4 Forest plot of Cox regression analysis for predicting the development of ITBL within 2 years post-LDLT. Last PELD score, GGT, RI, and LSM were the independent predictors. The hazard ratio for $\mathrm{RI}$ is 2.055 per 0.1 incremental increase.

Abbreviations: ITBL, ischemic-type biliary lesions; ALP, alkaline phosphatase; GGT, gamma-glutamyl transpeptidase; PSV, peak systolic velocity; RI, resistive index; LDLT, living-donor liver transplantation; PELD, last pediatric end-stage liver disease; LSM, liver stiffness measurement. 


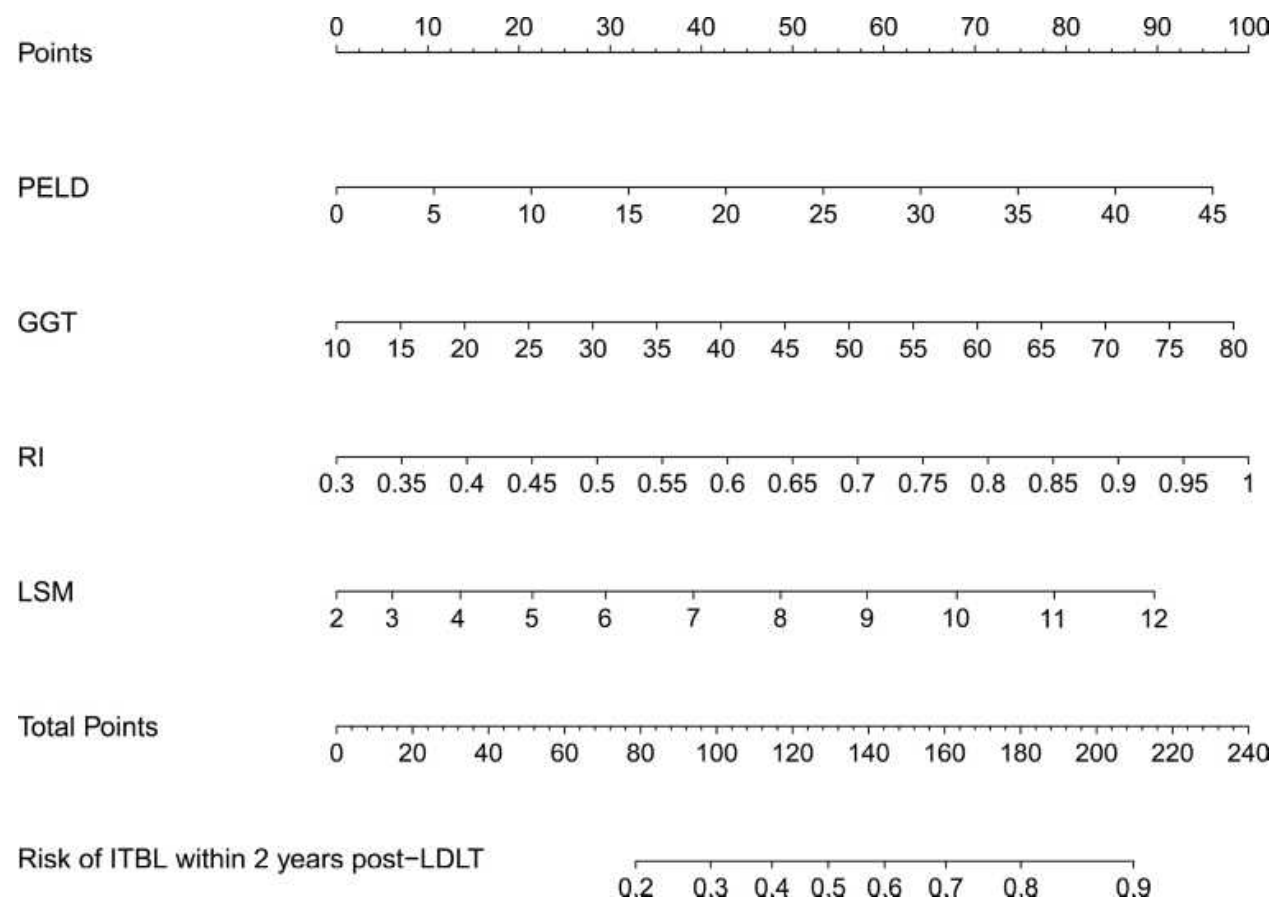

Figure 5 Nomogram predicting the risk of ITBL within 2 years post-LDLT. The top row showed the point assignment for each variable. Rows $2-5$ indicated the variables included in the nomogram. The bottom row showed the probability of ITBL within 2 years.

Abbreviations: ITBL, ischemic-type biliary lesions; LDLT, living-donor liver transplantation; GGT, gamma-glutamyl transpeptidase; PELD, last pediatric end-stage liver disease; RI, resistive index; LSM, liver stiffness measurement.

better performance than individual indicators for predicting the risk of ITBL within 2 years post-LDLT.

Most patients present symptoms of ITBL within the first year after liver transplantation. ${ }^{6,8,24}$ In our study, ITBL was diagnosed in 13 patients within one-year postLDLT and 5 were diagnosed within 1-1.5 years. Due to the anatomical limitations with tiny bile ducts, the occurrence of ITBL in LDLT is more common compared to deceased-donor liver transplantation despite good graft viability with short ischemia time. ${ }^{25}$ In this study, the incidence of ITBL within 2 years post-LDLT was $28.1 \%$, which was consistent with the reported incidence of biliary complications ( $28 \%$ to $32 \%$ post-LDLT). ${ }^{9}$ Although the etiology and risk factors for ITBL have been identified, a quick, convenient, and sensitive screening tool for early prediction of ITBL is limited.

PELD score has been shown to predict the prognosis of pediatric liver transplantation although it is designed to predict the risk of wait-list death before transplantation. ${ }^{26,27}$ In our study, the last PELD score prior to LDLT was significantly associated with the development of ITBL, according to the multivariate COX analysis. It suggested that the severity of liver dysfunction may be more important than the demographics and operative characteristics of pediatric recipients (eg, age, weight, cold ischemia time, etc) in affecting the post-LDLT outcomes. A study by $\mathrm{Oh}$ et $\mathrm{al}^{28}$ showed that increasing PELD scores were associated with graft loss and patient survival, which was similar to our findings. However, the ROC analysis of PELD in our study indicated an inaccurate prediction for ITBL. A similar conclusion was reached by Bourdeaux et al, ${ }^{29}$ in that high PELD children may not result in worsening outcomes.

As a more sensitive indicator of cholestasis, GGT will change earlier than other serological indicators after poor bile drainage. ${ }^{30}$ In the current study, GGT at onemonth post-LDLT was the independent predictor for ITBL. Some previous studies also reported a direct relationship between GGT and biliary complications. ${ }^{31,32}$ However, since cholestasis caused by ITBL is a chronic injury, it resulted in a slight elevation (within normal limits) in GGT at one-month post-LDLT. As indicated by the AUC of GGT, it contributed little predictive value to ITBL, which was consistent with the report by Que et al. ${ }^{33}$

Liver grafts were routinely monitored by ultrasoundbased multimodal imaging (gray-scale ultrasound, CDFI, 


\section{A}

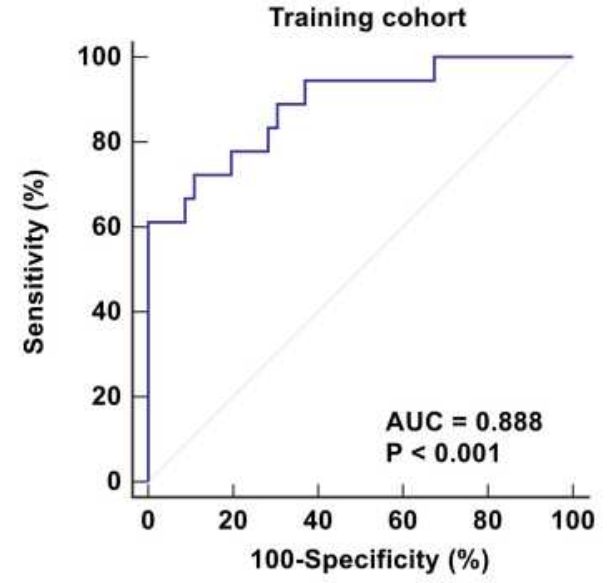

C

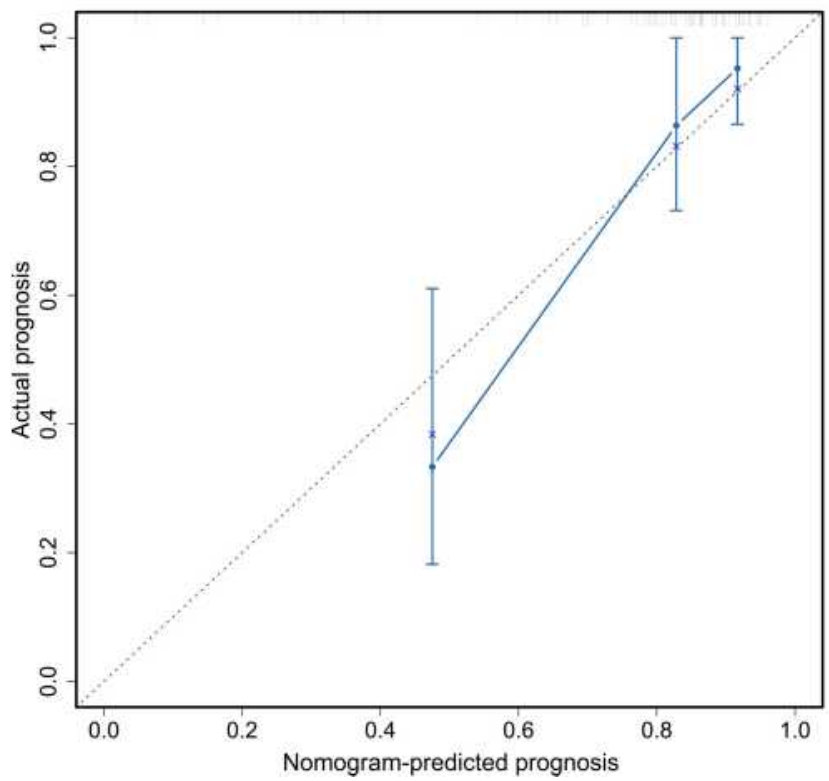

B

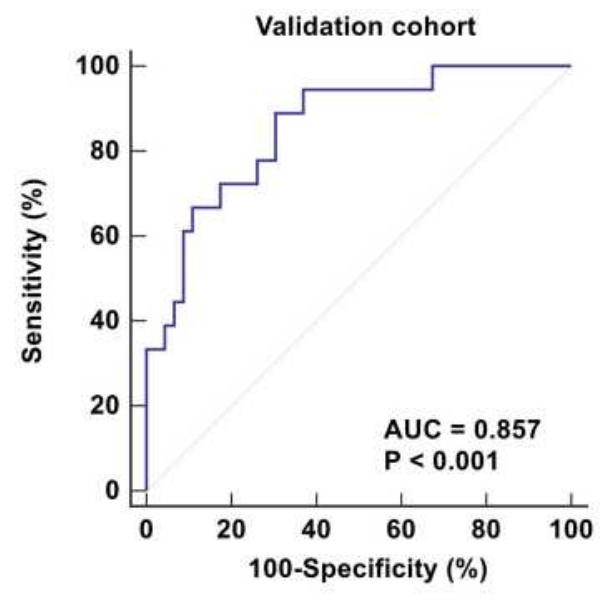

D

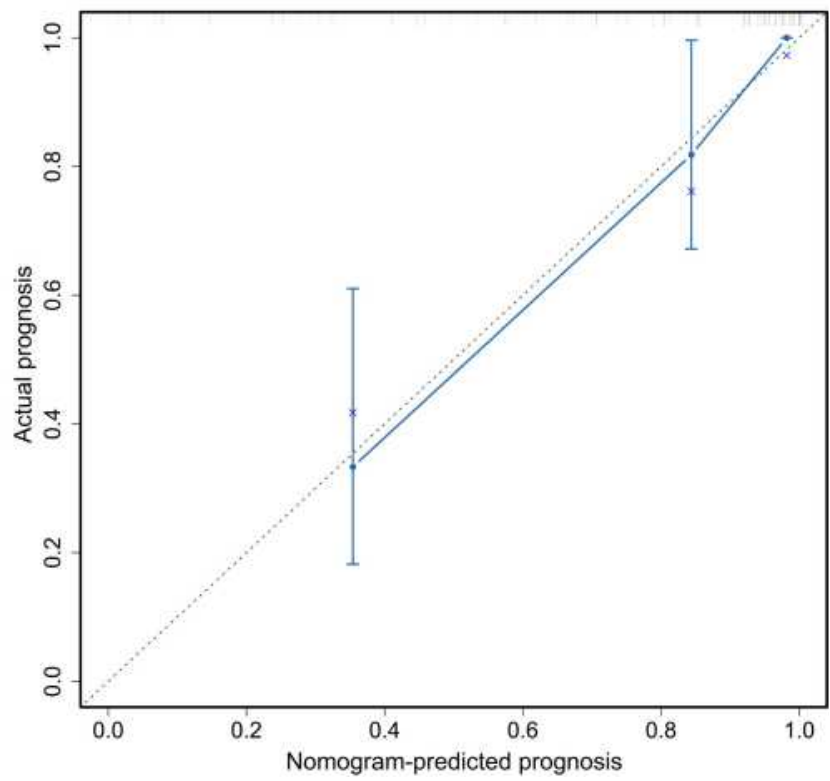

Figure 6 Internal and external validation of the nomogram predicting the risk of ITBL within 2 years post-LDLT. The ROC curves were plotted for the discrimination of the nomogram. The AUCs in the training cohort and validation cohort were 0.888 (95\% Cl 0.784 to 0.953$)$ (A) and 0.857 ( $95 \%$ Cl 0.748 to 0.932$)$ (B), respectively, indicating good discrimination. The calibration curves were plotted for evaluating the calibration of nomogram-predicted prognosis and actual prognosis estimated using KM analysis. The calibration plots in the training and validation datasets were shown in (C) and (D). Both indicated that the nomogram-predicted prognosis compared very well with the actual prognosis.

Abbreviations: ROC, receiver operating characteristic curve; AUC, area under the curve; 95\% Cl, 95\% confidence interval; KM, Kaplan-Meier.

SWE) after LDLT in our center. Since the blood supply to the biliary tract is solely dependent on arterial inflow, current preservation solutions and the difficulty in anastomosis between the graft and peribiliary vascular plexus may result in the disturbance of peribiliary plexus and subsequent damage of the biliary epithelium, eventually progressing to ITBL. ${ }^{6,34-36}$ Besides, the impaired bile secretion caused by ITBL may result in peribiliary fibrosis and intrahepatic cholestasis, leading to increased hydrostatic pressure in the biliary system. ${ }^{6}$ It will interfere with the conduction of ultrasound and show different stiffness in elastography. ${ }^{21,22}$ In our study, RI and LSM at one- month post-LDLT were proved to be associate with the ITBL, which indicated the alterations of hepatic vasculature and liver parenchymal elasticity in the early stage of ITBL. However, it is difficult to find during daily monitoring due to the minimal alterations (within normal limits). As indicated by the ROC analyses, they were not eligible for predicting the development of ITBL within 2 years post-LDLT as well.

Few prediction models are developed for the prediction of ITBL after LDLT currently. The advantage of our established nomogram is that all the predictors are convenient to obtain from routine monitoring. It could be utilized to 


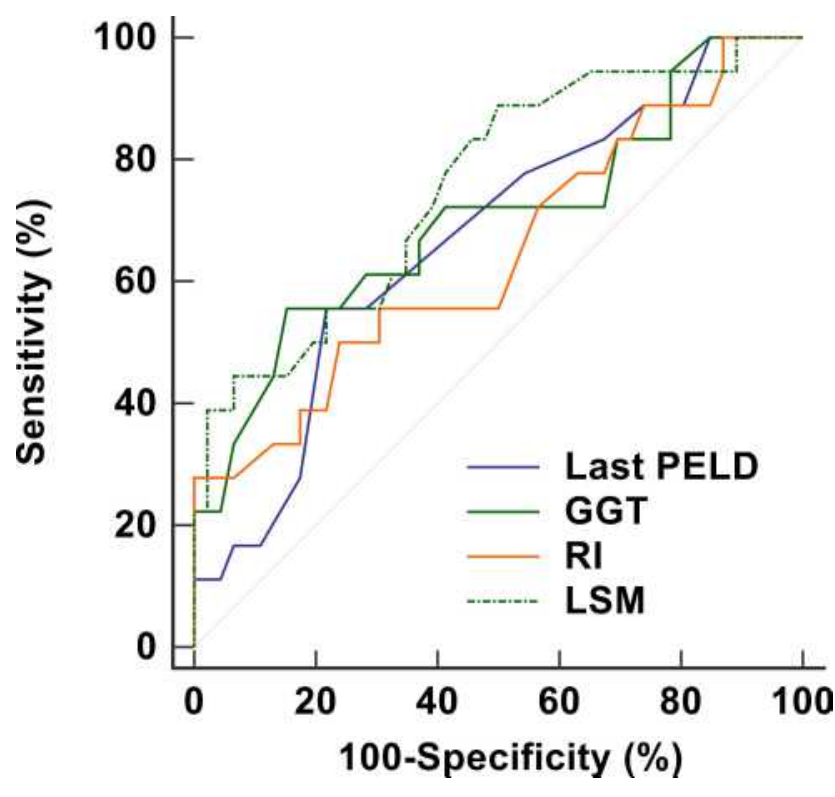

Figure 7 ROC curves of last PELD score, GGT, RI, and LSM in predicting the development of ITBL within 2 years post-LDLT. Their AUCs were $0.670(95 \% \mathrm{Cl}$ $0.54 \mathrm{I}$ to 0.783 ), 0.702 ( $95 \% \mathrm{Cl} 0.575$ to $0.8 \mathrm{I}$ ), 0.646 ( $95 \% \mathrm{Cl} 0.5 \mathrm{I} 7$ to 0.762 ), and 0.757 (95\% Cl 0.633 to 0.855$)$, respectively.

Abbreviations: ROC, receiver operating characteristic curve; PELD, last pediatric end-stage liver disease; GGT, gamma-glutamyl transpeptidase; RI, resistive index; LSM, liver stiffness measurement; ITBL, ischemic-type biliary lesions; AUC, area under the curve; $95 \% \mathrm{Cl}, 95 \%$ confidence interval.

calculate the scores corresponding to each independent predictor, and the predicted probability corresponding to the sum of the scores was the risk of IBTL within 2 years post-LDLT. The nomogram was verified good discrimination and calibration, which allowed a wise decision on preventive and therapeutic strategies. For example, the last PELD score prior to LDLT of a BA child was 15 , and his GGT, RI, and LSM value at one-month post-LDLT were $40 \mathrm{IU} / \mathrm{L}, 0.7$, and $7 \mathrm{kPa}$, respectively. The total score was about 160 , which indicated that the probability of ITBL within 2 years was about $70 \%$.

The main limitation of our study is the fact that the selection bias might present since the pediatric patients who were not eligible for the study were excluded at first. Besides, the nomogram was not validated in different populations. A further prospective investigation will help to validate our results on a larger scale to improve the performance of the constructed nomogram.

In conclusion, the indicators of liver function and ultrasound-based multimodal imaging at one-month post-LDLT are easy to obtain in the routine follow-up. The established nomogram may be useful for predicting the risk of ITBL within 2 years post-LDLT in pediatric patients with BA.

\section{Ethical Approval}

Ethical approval for the study was obtained from the ethics committee of Hunan Children's Hospital (HCHLL-202099).

\section{Informed Consent}

Informed consents were obtained from their parents or legal guardians.

\section{Acknowledgments}

The authors wish to thank all the study participants, research staff and students who participated in this work.

\section{Author Contributions}

Study design: Jin-qiao Liu, and Wen-juan Chen. Data collection and analysis: Jin-qiao Liu, Meng-jie Zhou, Wen-feng Li, and Ju Tang. Supervision: Jin-qiao Liu, and Wen-juan Chen. Statistics: Jin-qiao Liu, Wen-juan Chen, Meng-jie Zhou, Wen-feng Li, and Ju Tang. Manuscript writing: Jin-qiao Liu, Wen-juan Chen, Meng-jie Zhou, Wen-feng $\mathrm{Li}$, and Ju Tang. Manuscript revision: Jin-qiao Liu, and Wen-juan Chen. Approval of the manuscript: all authors. All authors made substantial contributions to conception and design, acquisition of data, or analysis and interpretation of data; took part in drafting the article or revising it critically for important intellectual content; agreed to submit to the current journal; gave final approval of the version to be published; and agree to be accountable for all aspects of the work.

\section{Funding}

Innovation Guidance Project of Hunan Provincial Science \& Technology Department (2020SK50515). Project of Hunan Provincial Health Commission (20209021301).

\section{Disclosure}

The authors declare that they have no conflicts of interest.

\section{References}

1. Kasahara M, Sakamoto S, Fukuda A. Pediatric living-donor liver transplantation. Semin Pediatr Surg. 2017;26(4):224-232. doi:10.10 53/j.sempedsurg.2017.07.008

2. Wang H, Jiang W, Zhou Z, Long J, Li W, Fan ST. Liver transplantation in mainland China: the overview of CLTR 2011 annual scientific report. Hepatobiliary Surg Nutr. 2013;2(4):188-197. doi:10.3978/j. issn.2304-3881.2013.08.09

3. Sharma S, Gurakar A, Jabbour N. Biliary strictures following liver transplantation: past, present and preventive strategies. Liver Transpl. 2008;14(6):759-769. doi:10.1002/lt.21509 
4. Sanchez-Urdazpal L, Gores GJ, Ward EM, et al. Ischemic-type biliary complications after orthotopic liver transplantation. Hepatology. 1992;16(1):49-53. doi:10.1002/hep.1840160110

5. Fisher A, Miller CM. Ischemic-type biliary strictures in liver allografts: the achilles heel revisited? Hepatology. 1995;21(2):589-591. doi:10.1002/hep.1840210245

6. Buis CI, Hoekstra H, Verdonk RC, Porte RJ. Causes and consequences of ischemic-type biliary lesions after liver transplantation. J Hepatobiliary Pancreat Surg. 2006;13(6):517-524. doi:10.1007/ s00534-005-1080-2

7. Wagner D, Bradatsch A, Kniepeiss D, et al. Incidence of ischemic type biliary lesions after liver transplantation using piggyback technique and retrograde reperfusion. Eur Surg. 2013;45(1):3-7. doi:10.1007/s10353-012-0166-8

8. Sanchez-Urdazpal L, Gores GJ, Ward EM, et al. Diagnostic features and clinical outcome of ischemic-type biliary complications after liver transplantation. Hepatology. 1993;17(4):605-609. doi:10.1002/ hep. 1840170413

9. Ryu CH, Lee SK. Biliary strictures after liver transplantation. Gut Liver. 2011;5(2):133-142. doi:10.5009/gnl.2011.5.2.133

10. Nakamura N, Nishida S, Neff GR, et al. Intrahepatic biliary strictures without hepatic artery thrombosis after liver transplantation: an analysis of 1113 liver transplantations at a single center. Transplantation. 2005;79(4):427-432. doi:10.1097/01.tp.0000152800.19986.9e

11. Ghinolfi D, De Simone P, Lai Q, et al. Risk analysis of ischemic-type biliary lesions after liver transplant using octogenarian donors. Liver Transpl. 2016;22(5):588-598. doi:10.1002/lt.24401

12. Zhang H, Shi Y, Wu H, et al. Change of hepatic arterial systolic/ diastolic ratio predicts ischemic type biliary lesion after orthotropic liver transplantation. Clin Imaging. 2016;40(3):419-424. doi:10.10 16/j.clinimag.2016.01.013

13. Guichelaar MM, Benson JT, Malinchoc M, Krom RA, Wiesner RH, Charlton MR. Risk factors for and clinical course of non-anastomotic biliary strictures after liver transplantation. Am J Transplant. 2003;3 (7):885-890. doi:10.1034/j.1600-6143.2003.00165.x

14. Verdonk RC, Buis CI, van der Jagt EJ, et al. Nonanastomotic biliary strictures after liver transplantation, part 2: management, outcome, and risk factors for disease progression. Liver Transpl. 2007;13 (5):725-732. doi:10.1002/lt.21165

15. Langrehr JM, Neuhaus R, Hinze R, et al. Incidence and etiologic factors for ischemic type biliary lesions after liver transplantation. Transplantation. 1999;67(7):S189. doi:10.1097/00007890-1999041 50-00756

16. Potthoff A, Hahn A, Kubicka S, et al. Diagnostic value of ultrasound in detection of biliary tract complications after liver transplantation. Hepat Mon. 2013;13(1):e6003-e6003. doi:10.5812/hepatmon.6003

17. Abdelaziz O, Attia H. Doppler ultrasonography in living donor liver transplantation recipients: intra- and post-operative vascular complications. World J Gastroenterol. 2016;22(27):6145-6172. doi:10.3748/wjg.v22.i27.6145

18. Goldschmidt I, Stieghorst H, Munteanu M, et al. The use of transient elastography and non-invasive serum markers of fibrosis in pediatric liver transplant recipients. Pediatr Transplant. 2013;17(6):525-534. doi:10.1111/petr.12116

19. Lee SH, Joo DJ, Kim SU, et al. Graft function measured by transient elastography in living donor liver transplantation: preliminary. Transplant Proc. 2013;45(8):3028-3031. doi:10.1016/j.transproceed. 2013.08.051

20. Bonini G, Pezzotta G, Morzenti C, Agazzi R, Nani R. Contrastenhanced ultrasound with SonoVue in the evaluation of postoperative complications in pediatric liver transplant recipients. J Ultrasound. 2007;10(2):99-106. doi:10.1016/j.jus.2007.02.008
21. Millonig G, Reimann FM, Friedrich S, et al. Extrahepatic cholestasis increases liver stiffness (FibroScan) irrespective of fibrosis. Hepatology. 2008;48(5):1718-1723. doi:10.1002/hep.22577

22. Trifan A, Sfarti C, Cojocariu C, et al. Increased liver stiffness in extrahepatic cholestasis caused by choledocholithiasis. Hepat Mon. 2011;11(5):372-375.

23. van Katwyk S, Coyle D, Cooper C, et al. Transient elastography for the diagnosis of liver fibrosis: a systematic review of economic evaluations. Liver Int. 2017;37(6):851-861. doi:10.1111/liv.13260

24. Campbell WL, Sheng R, Zajko AB, Abu-Elmagd K, Demetris AJ. Intrahepatic biliary strictures after liver transplantation. Radiology. 1994;191(3):735-740. doi:10.1148/radiology.191.3.8184054

25. Takatsuki M, Eguchi S, Kawashita Y, Kanematsu T. Biliary complications in recipients of living-donor liver transplantation. J Hepatobiliary Pancreat Surg. 2006;13(6):497-501. doi:10.1007/ s00534-005-1082-0

26. McDiarmid SV, Merion RM, Dykstra DM, Harper AM. Selection of pediatric candidates under the PELD system. Liver Transpl. 2004;10 (10 Suppl 2):S23-S30. doi:10.1002/lt.20272

27. Barshes NR, Lee TC, Udell IW, et al. The pediatric end-stage liver disease (PELD) model as a predictor of survival benefit and posttransplant survival in pediatric liver transplant recipients. Liver Transpl. 2006;12(3):475-480. doi:10.1002/lt.20703

28. Oh SH, Kim KM, Kim DY, et al. Long-term outcomes of pediatric living donor liver transplantation at a single institution. Pediatr Transplant. 2010;14(7):870-878. doi:10.1111/j.1399-3046.2010.01 357.x

29. Bourdeaux C, Tri TT, Gras J, et al. PELD score and posttransplant outcome in pediatric liver transplantation: a retrospective study of 100 recipients. Transplantation. 2005;79(9):1273-1276. doi:10.1097/ 00007890-200505150-00060

30. Siddique A, Kowdley KV. Approach to a patient with elevated serum alkaline phosphatase. Clin Liver Dis. 2012;16(2):199-229. doi:10. 1016/j.cld.2012.03.012

31. Ben-Ari Z, Weiss-Schmilovitz H, Sulkes J, et al. Serum cholestasis markers as predictors of early outcome after liver transplantation. Clin Transplant. 2004;18(2):130-136. doi:10.1046/j.1399-0012.2003. 00135.x

32. Shastri YM, Hoepffner NM, Akoglu B, et al. Liver biochemistry profile, significance and endoscopic management of biliary tract complications post orthotopic liver transplantation. World $J$ Gastroenterol. 2007;13(20):2819-2825. doi:10.3748/wjg.v13.i20. 2819

33. Que Y, Kaneko J, Sugawara Y, Tamura S, Makuuchi M. Role of protocol ultrasonography for detecting biliary stricture in adult living donor liver transplantation recipients. Biosci Trends. 2007;1 (1):62-65.

34. Li S, Stratta RJ, Langnas AN, Wood RP, Marujo W, Shaw BW Jr. Diffuse biliary tract injury after orthotopic liver transplantation. $\mathrm{Am}$ J Surg. 1992;164(5):536-540. doi:10.1016/s0002-9610(05)81196-1

35. Moench C, Moench K, Lohse AW, Thies J, Otto G. Prevention of ischemic-type biliary lesions by arterial back-table pressure perfusion. Liver Transpl. 2003;9(3):285-289. doi:10.1053/jlts.2003. 50015

36. Pirenne J, Van Gelder F, Coosemans W, et al. Type of donor aortic preservation solution and not cold ischemia time is a major determinant of biliary strictures after liver transplantation. Liver Transpl. 2001;7(6):540-545. doi:10.1053/jlts.2001.24641 


\section{Publish your work in this journal}

The International Journal of General Medicine is an international, peer-reviewed open-access journal that focuses on general and internal medicine, pathogenesis, epidemiology, diagnosis, monitoring and treatment protocols. The journal is characterized by the rapid reporting of reviews, original research and clinical studies across all disease areas. The manuscript management system is completely online and includes a very quick and fair peer-review system, which is all easy to use. Visit http://www.dovepress.com/ testimonials.php to read real quotes from published authors.

Submit your manuscript here: https://www.dovepress.com/international-journal-of-general-medicine-journal 